Western Washington University Western CEDAR

$3-1995$

\title{
Lazutkin Coordinates and Invariant Curves for Outer Billiards
}

\author{
Edoh Y. Amiran \\ Western Washington University, edoh.amiran@wwu.edu
}

Follow this and additional works at: https://cedar.wwu.edu/math_facpubs

Part of the Mathematics Commons

\section{Recommended Citation}

Amiran, Edoh Y., "Lazutkin Coordinates and Invariant Curves for Outer Billiards" (1995). Mathematics. 14.

https://cedar.wwu.edu/math_facpubs/14

This Article is brought to you for free and open access by the College of Science and Engineering at Western CEDAR. It has been accepted for inclusion in Mathematics by an authorized administrator of Western CEDAR. For more information, please contact westerncedar@wwu.edu. 


\title{
Lazutkin coordinates and invariant curves for outer billiards
}

\author{
Edoh Y. Amiran \\ Mathematics Department, Western Washington University, \\ Bellingham, Washington $98225-9063$
}

(Received 25 April 1994; accepted for publication 1 June 1994)

The outer billiard ball map (OBM) is defined from and to the exterior of a domain, $\Omega$, in the plane as taking a point, $q$, to another point, $q_{1}$, when the line segment with endpoints $q$ and $q_{1}$ is tangent to the boundary, $\partial \Omega$ (with a chosen orientation), and the point of tangency with the boundary divides the segment in half. Let $C$ be an invariant circle for the OBM on $\Omega$, with $\partial \Omega$ smooth with positive curvature. After computing the loss of derivatives between $\partial \Omega$ and $C$, it is shown via KAM theory that in this setting the OBM has uncountably many invariant circles in any neighborhood of the boundary. One is also led to an infinitesimal obstruction for the evolution property, an obstruction which, among closed smooth convex curves, is only removed for ellipses. (c) 1995 American Institute of Physics.

\section{INTRODUCTION}

This paper concerns smooth bounded planar domains whose boundary has strictly positive curvature. The outer billiard ball map (OBM) is defined from and to the exterior of such a domain as taking a point, $q$, to another point, $q_{1}$, when the line segment with endpoints $q$ and $q_{1}$ is tangent to the boundary, $\partial \Omega$ (with a chosen orientation), and the point of tangency with $\partial \Omega$ divides the segment in half.

An invariant circle for the OBM on a smooth convex domain is a smooth, strictly convex curve, $C$, homotopic to $\partial \Omega$ with the property that every point of $C$ is returned to $C$ under glancing reflection at the boundary. When any invariant circle for the OBM on any invariant circle is also an invariant circle for the boundary we say that the curves satisfy the evolution property.

Invariant circles for the outer billiard map were first discussed by Moser $^{1}$ where their existence far away from the boundary was proved. The general observation there is that the OBM is similar to the usual billiard map, with the advantage that the invariant curves for the OBM do not become singular as their distance from the boundary increases. It has been generally believed that the OBM and the billiard map have similar behavior, and indeed Tabachnikov ${ }^{2}$ has observed that the OBM is "dual" to the usual billiard map. Tabachnikov has also observed that Lazutkin's construction of invariant circles ${ }^{3}$ should apply. Lazutkin's estimates for the billiard ball map depend on the smoothness of a certain parameter for invariant curves (Mather's $H$ for twist maps). Here this parameter is analyzed directly, making the previous statement complete. The analysis also allows one to calculate expansions for the radius of curvature of an invariant circle directly, which yields a characterization of the transitivity property for the OBM. What is perhaps most interesting is that the smoothness of the invariant circles for the OBM is no better than that of the invariant circles for the usual billiard map, despite the better behavior of the former at infinity.

More technically, here coordinates which make direct use of the relation between a geometric criterion for the invariance of a closed convex curve and the rotation number of the OBM on the curve are used to show, via Lazutkin's theorem, ${ }^{3}$ that any sufficiently smooth boundary with positive curvature has uncountably many invariant circles in any neighborhood of the boundary. The same coordinates are used to calculate the relation between the curvatures of the boundary and of an invariant circle explicitly (following Ref. 4) and this relation leads to an infinitesimal obstruction for the evolution property. Among smooth curves with positive curvature this obstruction is only removed for ellipses. 


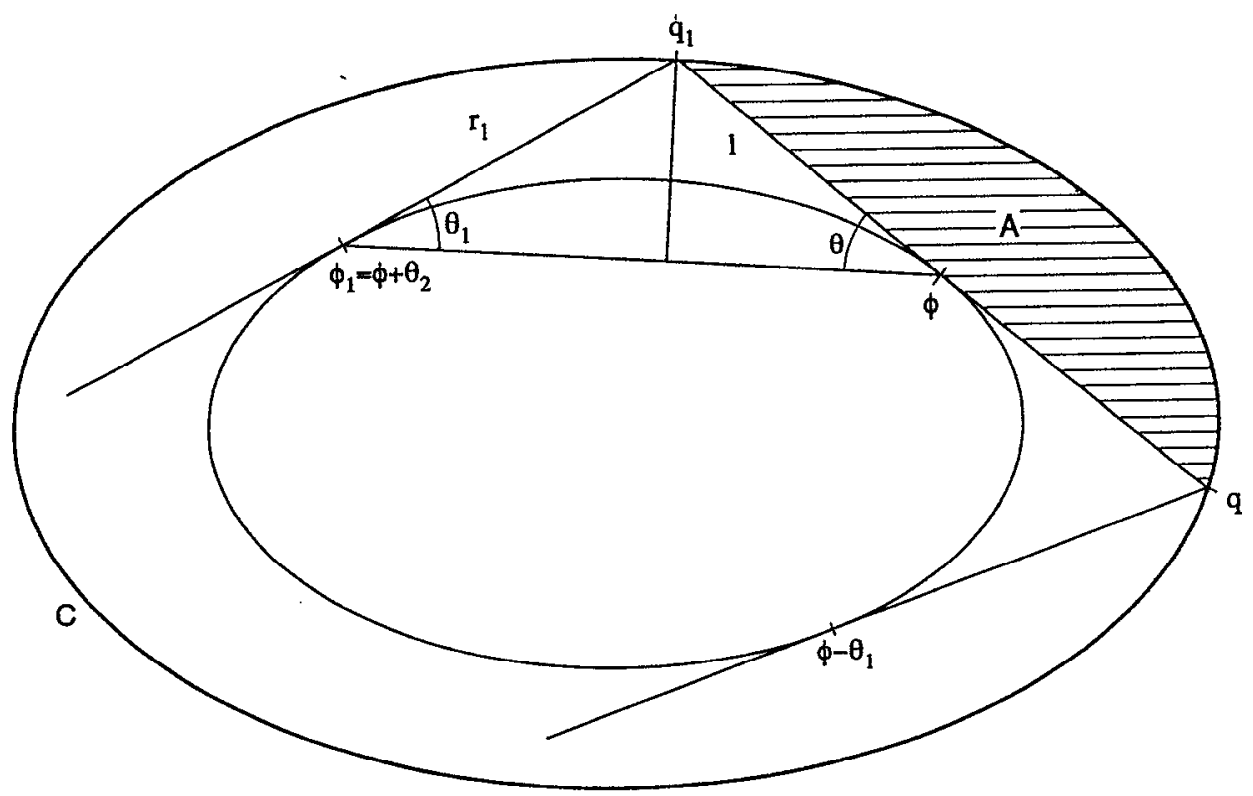

FIG. 1. $q_{1}=B(q)$ and the invariant curve $C$.

The main results are Theorems 3,5 , and 6 , which detail the relation between the curvature of an invariant circle and the curvature of the boundary, and formulate its consequences.

\section{THE OUTER BILLIARD MAP AND INVARIANT CURVES}

Let $\Omega \subset \mathbf{R}^{2}$ be a bounded open region with a smooth boundary, $\partial \Omega$, whose curvature is strictly positive. Then $\partial \Omega$ can be parametrized by tangent angle, $\phi$. Choosing the counterclockwise orientation and the $(1,0)$ direction as the reference for measuring the tangent angle, such regions are determined by their radius of curvature.

Let $q$ be a point in $\mathbf{R}^{2} \Omega \Omega$. Give $2 \Omega$ an orientation, say counterclockwise. Then there is a unique line through $q$ tangent to $\partial \Omega$, at some point $\phi$, so that the line segment $\overline{q \phi}$ has the direction of the tangent to $\partial \Omega$ at $\phi$. There is a unique $q_{1} \in \mathbf{R}^{2} \backslash \Omega$ with $q_{1} \neq q$ lying on the same tangent line and $\left|q_{1}-\phi\right|=|q-\phi|$ (the norm being calculated in $\mathbf{R}^{2}$ ). The outer billiard ball map, $B$, is defined as taking $q$ to $q_{1}$. See Fig. 1 for this definition, Definition 2.1, and the two lemmas of this section.

An invariant circle for the map $B$ is a closed smooth curve in $\mathbf{R}^{2} \backslash \Omega$ which is isotopic to $\partial \Omega$ and invariant under $B$. Such curves are characterized by Lemma 1 , where the area parameter, $A$, is the analogue of Lazutkin's parameter for billiards and Mather's $H$ for twist maps.

Lemma 1: A closed smooth curve $C$ in $\mathbf{R}^{2} \Omega$ is invariant under $B$ iff the area of the region not containing $\Omega$ and enclosed by $C$ and a chord tangent to $\partial \Omega$ at $\phi$ is independent of $\phi$.

Proof: Let $q, q_{1}$, and $\phi$ be as above and set $r=|q-\phi|$ and $l=\left|q_{1}-\phi\right|$. Denoting the area of the enclosed region by $A$,

$$
\frac{d A}{d \phi}=\frac{1}{2}\left(l^{2}-r^{2}\right)
$$


It is well known that $B$ preserves the Euclidean two-form. This is needed later for a theorem about the existence of invariant circles, so for completeness we include an elementary proof of this fact.

Proposition 2: $B$ preserves the Euclidean area.

Proof: Let points in $\mathbf{R}^{2} \backslash \Omega$ be described by coordinates $(\phi, r)$ as above. The corresponding Euclidean coordinates, $(x, y)$, are

$$
x=\int_{0}^{\phi} \chi(u) \cos (u) d u+r \cos (\phi), \quad y=\int_{0}^{\phi} \chi(u) \sin (u) d u+r \sin (\phi),
$$

from which one directly obtains $d x \wedge d y=r d \phi d r$.

Let $\left(\phi_{1}, r_{1}\right)=B(\phi, r)$, and let $l$ be as above. Define the functions $a$ and $b$ by $l=a\left(\phi, \phi_{1}\right)$ and $r_{1}=b\left(\phi, \phi_{1}\right)$.

Since $l=r$,

$$
d r_{1} \wedge d \phi_{1}=-\frac{\partial b}{\partial \phi}{\frac{\partial a}{\partial \phi_{1}}}^{-1} d l \wedge d \phi .
$$

Denote the $\mathbf{R}^{2}$-coordinates of the points $\phi$ and $\phi_{1}$ (on $\left.\partial \Omega\right)$ by $(x, y)$ and $\left(x_{1}, y_{1}\right)$, respectively. Then

$$
x+l \cos (\phi)=x_{1}-r_{1} \cos \left(\phi_{1}\right) \text { and } y+l \sin (\phi)=y_{1}-r_{1} \sin \left(\phi_{1}\right),
$$

from which

$$
a\left(\phi, \phi_{1}\right)=\frac{-\left(y_{1}-y\right) \cos \left(\phi_{1}\right)+\left(x_{1}-x\right) \sin \left(\phi_{1}\right)}{\sin \left(\phi_{1}-\phi\right)}
$$

and

$$
b\left(\phi, \phi_{1}\right)=\frac{-\left(x_{1}-x\right) \sin (\phi)+\left(y_{1}-y\right) \cos (\phi)}{\sin \left(\phi_{1}-\phi\right)} .
$$

Denoting the angle in $\mathbf{R}^{2}$ between the segment $\phi \phi_{1}$ and $\partial \Omega$ at $\phi$ by $\theta$ and the angle between $\phi \phi_{1}$ and $\partial \Omega$ at $\phi_{1}$ by $\theta_{1}$, a direct calculation shows that

$$
-\frac{\partial b}{\partial \phi}{\frac{\partial a}{\partial \phi_{1}}}^{-1}=\frac{\sin \theta_{1}}{\sin \theta}
$$

Directly from the geometry one observes that $l \sin \theta=r_{1} \sin \theta_{1}$, which completes the proof.

\section{RADII OF CURVATURE}

Definition 3.1: For $C \subset \mathbf{R}^{2} \backslash \Omega$ an invariant circle for the outer billiard map, $B$, on $\lambda \Omega$, we say that $\partial \Omega$ is the A-involute of $\mathbf{C}$ if the area bounded by $C$ and a chord to $\partial \Omega$ (as described in Lemma 1) is $A$. We call $A$ the area parameter for $C$.

Let $\chi$ denote the radius of curvature of $C$ and $\rho$ denote the radius of curvature of $2 \Omega$, both given in terms of tangent angle [with respect to a fixed direction, say $(1,0)]$. Assume that $C$ is an invariant curve for $B$. Then $\rho$ can be obtained from $\chi$ and the area parameter $A$ using the geometric description from Sec. II. We now examine this calculation.

Fix a value, $\phi$, for the tangent angle, and consider the chord tangent to $\partial \Omega$ at $\phi$. This chord meets the invariant circle $C$ at $\phi-\theta_{1}$ and at $\phi+\theta_{2}$ (by convention both thetas are positive). 
Choose $x-y$ coordinates (in $\mathbf{R}^{2}$ ) so that the $x$ axis is along the chord, the origin is at $\phi-\theta_{1}$, and the $y$ axis is perpendicular to the chord and oriented so that points in the region with area $A$ have a positive $y$ coordinates. Then in terms of $\chi$

$$
x(\phi)=\int_{\phi-\theta_{1}}^{\phi} \chi(u) \cos (u) d u, \quad y(\phi)=\int_{\phi-\theta_{1}}^{\phi} \chi(u) \sin (u) d u,
$$

and using Stokes' theorem to calculate the area $\left(A=\int x d y\right)$,

$$
A=-\int_{-\theta_{1}}^{\theta_{2}} \chi(\phi+v) \cos (v)\left(\int_{-\theta_{1}}^{v} \chi(\phi+u) \sin (u) d u\right) d v .
$$

Moreover, since $y=0$ along the chord,

$$
\int_{-\theta_{1}}^{\theta_{2}} \chi(\phi+u) \sin (u) d u=0 .
$$

Using (3.2) we solve formally for $\theta_{2}$ as a function of $\theta_{1}$ :

$$
\begin{aligned}
\theta_{2} \sim \theta_{1}-\frac{2}{3} \chi^{-1} \chi^{\prime} \theta_{1}^{2}+\frac{4}{9} \chi^{-2}\left(\chi^{\prime}\right)^{2} \theta_{1}^{3}+\left(-\frac{2}{45} \chi^{-1} \chi^{\prime}-\frac{16}{27} \chi^{-3}\left(\chi^{\prime}\right)^{3}+\frac{1}{3} \chi^{-2} \chi^{\prime} \chi^{\prime \prime}-\frac{1}{15} \chi^{-1} \chi^{(3)}\right) \theta_{1}^{4} \\
+\left(\frac{4}{45} \chi^{-2} \chi^{\prime}+\frac{64}{81} \chi^{-4}\left(\chi^{\prime}\right)^{3}-\frac{2}{3} \chi^{-3} \chi^{\prime} \chi^{\prime \prime}+\frac{2}{15} \chi^{-2} \chi^{(3)}\right) \theta_{1}^{5}+O\left(\theta^{6}\right),
\end{aligned}
$$

where each $\chi$ above is evaluated at $\phi$.

Then using the above in (3.1), the area has the expansion

$$
A \sim \frac{2}{3} \chi^{2} \theta_{1}^{3}-\chi \chi^{\prime} \theta_{1}^{4}+\left(-\frac{2}{15} \chi^{2}+\frac{53}{90}\left(\chi^{\prime}\right)^{2}+\frac{4}{15} \chi \chi^{\prime \prime}\right) \theta_{1}^{5}+O\left(\theta^{6}\right) .
$$

We invert this last relation to obtain

$$
\theta_{1} \sim\left(\frac{3}{2}\right)^{1 / 3} \chi^{-2 / 3} A^{1 / 3}+\left(\frac{3}{2}\right)^{2 / 3} \frac{1}{2} \chi^{-7 / 3} \chi^{\prime} A^{2 / 3}+\left(\frac{3}{2}\right)\left(\frac{1}{15} \chi^{-2}+\frac{41}{90} \chi^{-4}\left(\chi^{\prime}\right)^{2}-\frac{2}{15} \chi^{-3} \chi^{\prime \prime}\right) A+O\left(A^{4 / 3}\right)
$$

To calculate the radius of curvature of the boundary, $\rho$, we use the distances, $r$ and $l$, of $\phi-\theta_{1}$ and $\phi+\theta_{2}$ from $\phi$. Then

$$
\begin{aligned}
& \int_{0}^{\phi} \rho(u) \cos (u) d u-r \cos (\phi)=x_{0}+\int_{0}^{\phi-\theta_{1}} \chi(u) \cos (u) d u, \\
& \int_{0}^{\phi} \rho(u) \sin (u) d u-r \sin (\phi)=y_{0}+\int_{0}^{\phi-\theta_{1}} \chi(u) \sin (u) d u, \\
& \int_{0}^{\phi} \rho(u) \cos (u) d u+l \cos (\phi)=x_{0}+\int_{0}^{\phi+\theta_{2}} \chi(u) \cos (u) d u,
\end{aligned}
$$

and

$$
\int_{0}^{\phi} \rho(u) \sin (u) d u+l \sin (\phi)=y_{0}+\int_{0}^{\phi+\theta_{2}} \chi(u) \sin (u) d u
$$

Using these and $r=l$ we obtain 


$$
\rho(\phi)=\frac{1}{2}\left(r \cot \left(\theta_{1}\right)+l \cot \left(\theta_{2}\right)\right)
$$

and for $r$ and $l$

$$
r(\phi)=\chi\left(\phi-\theta_{1}\right) \sin \left(\theta_{1}\right)\left[1-\frac{d \theta_{1}}{d \phi}\right]
$$

and

$$
l(\phi)=\chi\left(\phi+\theta_{2}\right) \sin \left(\theta_{2}\right)\left[1+\frac{d \theta_{2}}{d \phi}\right]
$$

Replacing the expressions for $r$ and $l$ in (3.6) and using (3.3) and (3.5), we have

$$
\begin{aligned}
\rho \sim & \chi+\left(\frac{3}{2}\right)^{2 / 3}\left(-\frac{1}{2} \chi^{-1 / 3}-\frac{2}{9} \chi^{-7 / 3}\left(\chi^{\prime}\right)^{2}+\frac{1}{6} \chi^{-4 / 3} \chi^{\prime \prime}\right) A^{2 / 3}+\left(\frac{3}{2}\right)^{4 / 3}\left(-\frac{1}{40} \chi^{-5 / 3}-\frac{11}{90} \chi^{-11 / 3}\left(\chi^{\prime}\right)^{2}\right. \\
& -\frac{77}{405} \chi^{-17 / 3}\left(\chi^{\prime}\right)^{4}+\frac{1}{20} \chi^{-8 / 3} \chi^{\prime \prime}+\frac{77}{270} \chi^{-14 / 3}\left(\chi^{\prime}\right)^{2} \chi^{\prime \prime}-\frac{2}{45} \chi^{-11 / 3}\left(\chi^{\prime \prime}\right)^{2}-\frac{1}{15} \chi^{-11 / 3} \chi^{\prime} \chi^{(3)} \\
& \left.+\frac{1}{120} \chi^{-8 / 3} \chi^{(4)}\right) A^{4 / 3}+O\left(A^{5 / 3}\right)
\end{aligned}
$$

Here $\rho$ and $\chi$ and the derivatives of $\chi$ are all evaluated at $\phi$.

All calculations above were checked by hand, but the calculations involving power series were also carried out using Mathematica.

As a result of the calculations above we are in position to show the following.

Theorem 3: Assume that the boundary of a table for the outer billiard map is smooth with radius of curvature $\rho>0$ and is the $A$-involute of the invariant circle $C$ with radius of curvature $\chi$.

Then if $\chi$ is viewed as fixed, $\rho$ is a smooth function of $A^{2 / 3}$ and the coefficient of $A^{2 n / 3}$ in the expansion of $\rho(A)$ is an ordinary differential operator in $\chi$ of order exactly $2 n$.

Proof: Equations (3.2) and (3.3) and the implicit function theorem show that $\theta_{2}$ is a smooth function of $\theta_{1}$. Then (3.1) and (3.4) and the implicit function theorem show that $\theta_{1}$ is a smooth function of $A^{1 / 3}$, and (3.6) and (3.7) show that $\rho$ is a smooth function of $A^{1 / 3}$. Moreover, changing the orientation of the table would change the roles of $\theta_{1}$ and $\theta_{2}$ and hence, in the equations above, change their signs. In particular, this would make $A$ negative in (3.4). However, a change of orientation would not change $\rho$ or $\chi$ so the series for $\rho$ must be even in $A^{1 / 3}$.

It is clear that the highest derivative appearing in the expansions obtained from (3.6) and (3.7) is the highest derivative in the expansions for $\chi\left(\phi-\theta_{1}\right)$ and $\chi\left(\phi+\theta_{2}\right)$ in (3.6) and that this derivative does not cancel in (3.7) because $\theta_{2} \sim \theta_{1}+O\left(\theta_{1}^{2}\right)$ and because of the $d \theta_{j} / d \phi$ terms having opposite signs in (3.7). Hence if we truncate the series after the terms involving $A^{2 n / 3}$, the coefficient in the expansion of $\rho(A)$ with the highest derivative is an ordinary differential operator in $\chi$ of order exactly $2 n$.

This theorem is used in Sec. IV.

\section{THE AREA PARAMETER AND THE ROTATION NUMBER}

The main objective of this section is to show that there is a smooth coordinate change on the outer billiard table, to coordinates $(\xi, \eta)$, so that for each poorly approximated rotation number in a neighborhood of 0 there is an invariant circle with that rotation number, and each such curve has $\eta=$ constant on it. In other words, one can "construct" invariant circles using KAM techniques (as in Refs. 3 and 5) so that the coordinates depend smoothly on the rotation number.

The rotation number is, essentially, $\theta_{1}+\theta_{2}$ of Sec. III, and the smoothness follows from the computations there and from the role that the area $A$ plays in characterizing the invariant circles (Lemma 1). The remainder of this section supplies the justification for these (yet vague) statements. 
Let $S^{1}$ denote the circle with unit radius and $f: S^{1} \rightarrow S^{1}$ be a homeomorphism isotopic to the identity. Then the rotation number of $f$ at $\phi \in S^{1}$ is

$$
\rho(\phi)=\frac{1}{2 \pi} \lim _{n \rightarrow \infty} \frac{1}{n} \sum_{i=1}^{n}\left|f^{i}(\phi)-f^{i-1}(\phi)\right|,
$$

where the distance is measured by tangent angle along $S^{1}$ with a fixed orientation.

Poincare showed that for maps of the circle the rotation number is well defined (Ref. 6 or 7) and independent of the starting point $\phi$ (see section 6 of Ref. 7).

When $\partial \Omega$ is twice differentiable and has positive curvature and $C$ is an invariant circle for the OBM, the OBM restricted to $C$ is diffeomorphic to a map on the circle (through the identification of points with the same tangent angles) and thus has a rotation number that is independent of the starting point on $C$. (Since in absolute value the rotation number of a map on the circle and its inverse are the same, ${ }^{6}$ the rotation number is independent of the choice of orientations.)

Since the rotation number is the same for all points in $C$, it is equal to its average. Let $\left(\phi^{\prime}, r^{\prime}\right)=B(\phi, r)$ and let $\theta_{j}(\phi)$ be the angles formed with the invariant circle, $C$, by the chord tangent to the boundary at $\phi$. Then,

$$
\eta=\frac{1}{2 \pi} \int_{0}^{2 \pi}\left(\theta_{2}(\phi)+\theta_{1}\left(\phi^{\prime}\right)\right)=\frac{1}{2 \pi} \int_{0}^{2 \pi}\left(\theta_{2}(\phi)+\theta_{1}(\phi)\right) .
$$

However, $\theta_{j}$ are smooth functions of $A^{1 / 3}$, and, after substituting $\rho+O\left(A^{2 / 3}\right)$ for $\chi$ in (3.5) and then in (3.3), we have

$$
\eta \sim \frac{1}{\pi}\left(\frac{3}{2}\right)^{2 / 3}\left(\int_{0}^{2 \pi} \rho^{-2 / 3}(\phi) d \phi\right) A^{1 / 3}+O(A) .
$$

In fact, (3.1) and (3.2) show that the coefficients of the term $A^{m / 3}$ in the expansions for $\theta_{j}$ depend on, at most, $m$ derivatives of $\chi$. By Theorem $3, \chi$ is smooth in $A^{2 / 3}$ with coefficients of $A^{2 m / 3}$, depending on at most $2 m$ derivatives of $\chi$. Thus (4.2) and the implicit function theorem establish the following theorem.

Theorem 4: Assume that the boundary of a table for the outer billiard map is smooth with radius of curvature $\rho>0$ and is the $A$-involute of the invariant circle $C$ with radius of curvature $\chi$.

Then if $\chi$ is viewed as fixed, $\rho$ is a smooth function of $\eta^{2}$ and the coefficient of $\eta^{2 n}$ in the expansion of $\rho$ is an ordinary differential operator in $\chi$ of order exactly $2 n$.

Now the series for $\rho$ in powers of $\eta^{2}$ with coefficients depending on $\chi$ and its derivatives can be truncated and inverted, yielding an error of the type $O\left(\eta^{2 k}\right)$ for any desired $k$ and depending on finitely many, say $T$, derivatives of $\rho$. Say

$$
\chi_{k}(\phi) \sim \rho(\phi)+\sum_{m=1}^{k} C_{m}(\rho) \eta^{2 m}
$$

The coordinate $\phi$ is defined on the entire outer billiard table and

$$
(v, w)=\left(\int_{0}^{\phi} \chi_{k}(\phi) \cos (\phi), \int_{0}^{\phi} \chi_{k}(\phi) \sin (\phi)\right)
$$

are coordinates with $T$ fewer derivatives than $\rho$, and on an invariant circle, they are the original $(x, y)$ coordinates, up to an $O\left(\eta^{2 k+2}\right)$ error. These coordinates are smooth in $\eta$. 
Lazutkin used KAM theory to show that the error above can be corrected near a family of invariant circles. His result, which transfers exactly to our setting in view of Theorem 4 , is the following.

Corollary 5: When $\rho$ is $C^{r}$ with

$$
r>3+(3 \sigma+6)\left(6 k+9+\frac{4(k+2)^{3}}{2 k+3}\right)
$$

and $\sigma>3$, there is, with a chosen constant $0<a<1$, a $C^{k}$ system of coordinates $(\xi, \nu)$ defined for $0 \leqslant \nu \leqslant a$ so that

(1) $\nu=0$ is the boundary of the outer billiard table,

(2) $\xi$ is periodic with period $2 \pi$, and

(3) for any $0 \leqslant \eta \leqslant a$ with

$$
|n \eta-m|>a|m| n^{0.5-\sigma}
$$

the curve $\nu=\eta$ is an invariant circle for the OBM.

\section{CALCULATION OF AN EQUATION FOR THE TRANSITIVITY PROPERTY}

Let $\rho$ denote the radius of curvature of $2 \Omega$. Assume that for each $A$ in some nonempty interval $[0, T]$, there is an invariant curve with radius of curvature $\chi$ s.t. $\rho=V(A, \chi)$. Also assume that for each $A \in[0, T]$ and $n \geqslant 0$ there are $E_{n}=E(A, n), F_{n}=F(A, n)$, and $\nu_{n}=\nu(A, n)$ with

$$
V(A, \chi)=\rho, \quad V\left(E_{n}, \chi\right)=\nu_{n}, \quad \text { and } \quad V\left(F_{n}, \nu_{n}\right)=\rho,
$$

so that in addition $E_{0}=R, F_{0}=0$, and as $n \rightarrow \infty E_{n} \rightarrow 0$ while $F_{n} \rightarrow A\left(\nu_{n} \rightarrow \chi\right.$ as $\left.n \rightarrow \infty\right)$. Such a curve, $\partial \Omega$, is said to satisfy the transitivity property for the OBM.

A curve with the transitivity property has an integrable OBM; an invariant circle passes through every point sufficiently near the boundary. In contrast, Gutkin and Katok ${ }^{8}$ have shown that a curve with $\rho=0$ at some point is not integrable.

Recall from Theorem 3 that

$$
V(A, \chi) \sim \sum_{j=0}^{\infty} V_{j}(\chi) A^{2 j / 3},
$$

with $V_{j}(\chi)$ an ordinary differential operator. We also know that $V(0, \chi)=\chi$ so $V_{0}$ is the identity.

Interpreting $V\left(E_{n}, V\left(F_{n}, \chi\right)\right)=V(A, \chi)$ in the sense of power series, we obtain

$$
\begin{aligned}
& V_{0}(\chi)+\left(V_{1}(\chi)\right)\left(E_{n}^{2 / 3}+F_{n}^{2 / 3}\right)+\left(V_{1}^{\mathrm{two}}(\chi)\right) E_{n}^{2 / 3} F_{n}^{2 / 3}+\left(V_{2}(\chi)\right)\left(E_{n}^{4 / 3}+F_{n}^{4 / 3}\right) \\
& =V_{0}(\chi)+V_{1}(\chi) A^{2 / 3}+V_{2}(\chi) A^{4 / 3}+O\left(A^{6 / 3}\right),
\end{aligned}
$$

where $V_{1}^{\text {two }}(\chi)$ is the coefficient of $A^{2 / 3}$ in $V_{1}\left(\chi+V_{1}(\chi) A^{2 / 3}\right)$.

As $n$ approaches infinity,

$$
A^{2 / 3} \sim E_{n}^{2 / 3}+F_{n}^{2 / 3}+E_{n}^{2 / 3} g(k)\left(a E_{n}^{2 / 3}+b F_{n}^{2 / 3}\right)+O\left(A^{6 / 3}\right) .
$$

Thus (5.1) with $A$ approaching 0 yields $a=0$ and

$$
V_{1}^{\text {two }}(\chi(A))=V_{1}(\chi(A)) G(\chi(A))+2 V_{2}(\chi(A)) \quad[G(\chi)=b g(\chi)] .
$$


Above, $g$ and $G$ depend on the curve, but are independent of the tangent angle, and the radius of curvature is evaluated at the same point (tangent angle).

Since as $A \rightarrow 0, \chi(A) \rightarrow \rho$,

$$
V_{1}^{t w o}(\rho)-2 V_{2}(\rho)=G(\rho) V_{1}(\rho)
$$

After solving for $V_{1}$ and $V_{2}$ from the geometric description (see Sec. III) and calculating $V_{1}^{\text {two }}$, this equation becomes

$$
\begin{gathered}
\left(\frac{3}{2}\right)^{4 / 3} \rho^{-5 / 3}\left(\frac{1}{90} \rho^{-1} \rho^{(4)}-\frac{4}{45} \rho^{-2} \rho^{\prime} \rho^{(3)}-\frac{8}{135} \rho^{-2}\left(\rho^{\prime \prime}\right)^{2}+\frac{154}{405} \rho^{-3}\left(\rho^{\prime}\right)^{2} \rho^{\prime \prime}+\frac{1}{15} \rho^{-1} \rho^{\prime \prime}-\frac{308}{1215} \rho^{-4}\left(\rho^{\prime}\right)^{4}\right. \\
\left.-\frac{22}{135} \rho^{-2}\left(\rho^{\prime}\right)^{2}-\frac{1}{30}\right)=G(\rho)\left(\frac{3}{2}\right)^{2 / 3} \rho^{-1 / 3}\left(\frac{1}{6} \rho^{-1} \rho^{\prime \prime}-\frac{2}{9} \rho^{-2}\left(\rho^{\prime}\right)^{2}-\frac{1}{2}\right)
\end{gathered}
$$

Equation (5.3) must be satisfied by any $C^{5}$ convex curve on which the OBM has the transitivity property. It is satisfied by the radius of curvature of any ellipse and its rotations.

We are set to complete the proof the following theorem.

Theorem 6: The only closed planar curves with positive curvature that satisfy the transitivity property for the outer billiard map are ellipses.

Proof: We have a fourth-order differential equation in the radius of curvature with one undetermined parameter $(G)$. The solutions in which we are interested represent closed curves, so two conditions must be satisfied (one for each coordinate). Thus, loosely speaking, we obtain a threeparameter family of solutions which match with ellipses and their rotations. We need to show that the closure conditions are not automatically satisfied.

More precisely we argue as follows. Notice that the left-hand side of (5.3) is homogeneous of degree $-5 / 3$ under dilations of $\mathbf{R}^{2}$ (i.e., scaling of the radius of curvature), while the right-hand side is homogeneous of degree $-1 / 3$. It is clear from (5.2) that $G>0$, so we may assume that the region is so scaled that $G(w)=(3 / 2)^{2 / 3} / 15$ (this number is chosen so that circles are represented by the unit circle). Next note that if a curve is closed and its radius of curvature is positive, then its radius of curvature is bounded below by a positive number, and thus a solution to Eq. (5.3) develops no singularities as $\phi$ increases. It follows that the solutions to (5.3) representing such curves vary smoothly with the initial conditions.

Since we are only interested in closed curves, we may assume that the curve has a maximum at tangent angle 0 , so $\rho^{\prime}(0)=0$ [and $\left.\rho^{\prime \prime}(0) \leqslant 0\right]$. Retaining the assumption that $G(\rho)=(3 / 2)^{2 / 3} / 15$, we are left with three initial conditions, $\rho(0), \rho^{\prime \prime}(0)$, and $\rho^{(3)}(0)$. For any (positive) value of $\rho(0)$, say, there is an ellipse with that radius of curvature at its minimum and $G=(3 / 2)^{2 / 3} / 15$.

Since the solution varies smoothly with initial conditions, it suffices to fix $\rho(0)$ and vary $\rho^{\prime \prime}(0)$ and $\rho^{(3)}(0)$ from those of the ellipse to check that the resulting curves are not closed (their radius of curvature is not periodic with period $2 \pi$ ). This will show that the closure conditions are not automatically satisfied.

Numerically calculated solutions (see the end of this section) show that when either $\rho^{\prime \prime}(0)$ or $\rho^{(3)}(0)$ are varied the solution does not have period $2 \pi$ and that, moreover, when $\rho^{\prime \prime}(0)$ is varied $\rho^{\prime}(0)$ and $\rho^{(3)}(0)$ move in the same direction while when $\rho^{(3)}(0)$ is varied $\rho^{\prime}(0)$ and $\rho^{(3)}(0)$ move in opposite directions. Thus varying $\rho^{\prime \prime}(0)$ and $\rho^{(3)}(0)$ simultaneously still results in a solution that represents a curve which is not closed. Since there are no singularities, there are no isolated solutions and this completes the proof.

In satisfying $G=(3 / 2)^{2 / 3} / 15$ we use the fact that for an ellipse with major axis $2 a$ and minor axis $2 b$ the radius of curvature is

$$
a^{2} b^{2}\left(a^{2} \sin (\phi)^{2}+b^{2} \cos (\phi)^{2}\right)^{-3 / 2},
$$

and the constant is 


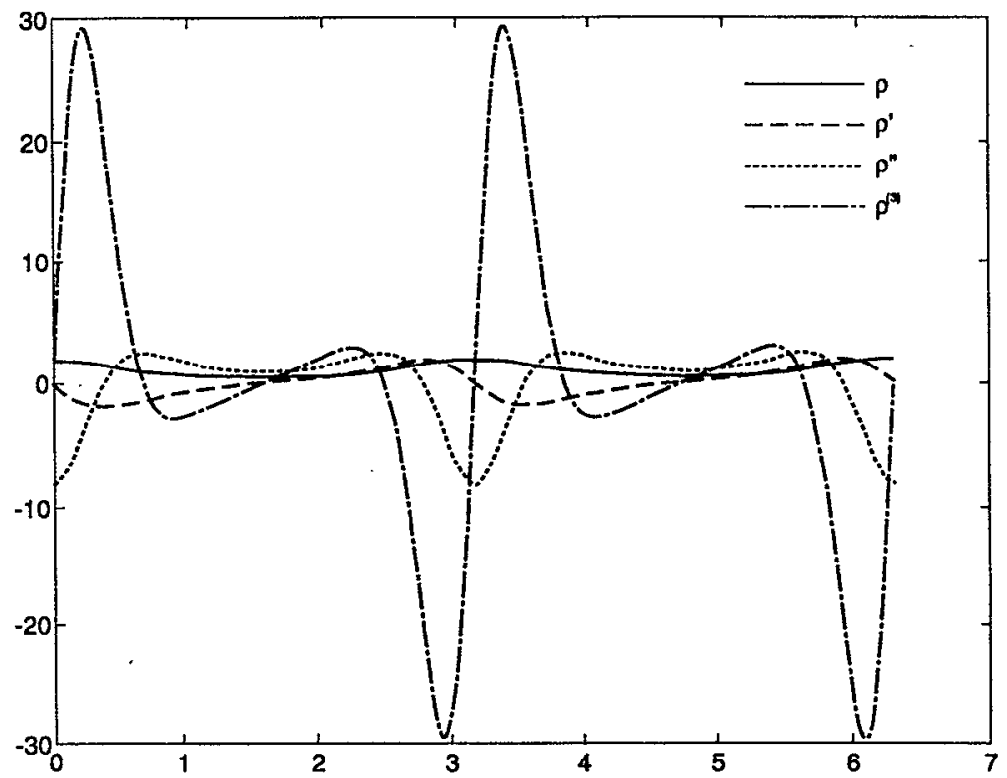

FIG. 2. An ellipse.

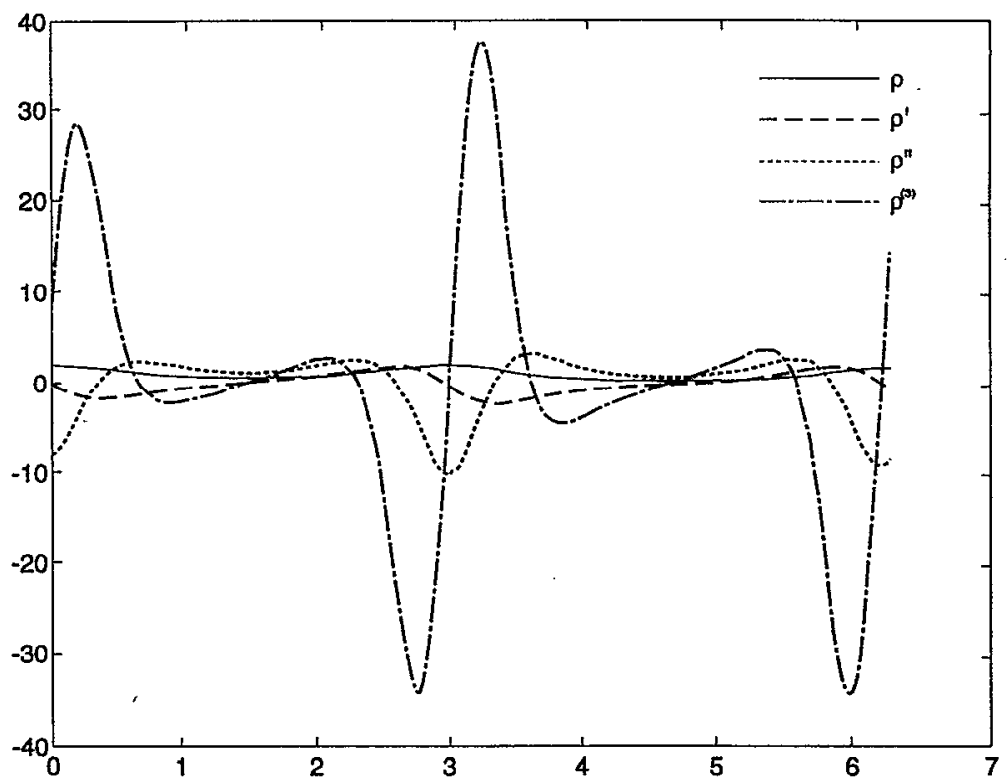

FIG. 3. An increase in $\rho^{(3)}(0)$. 


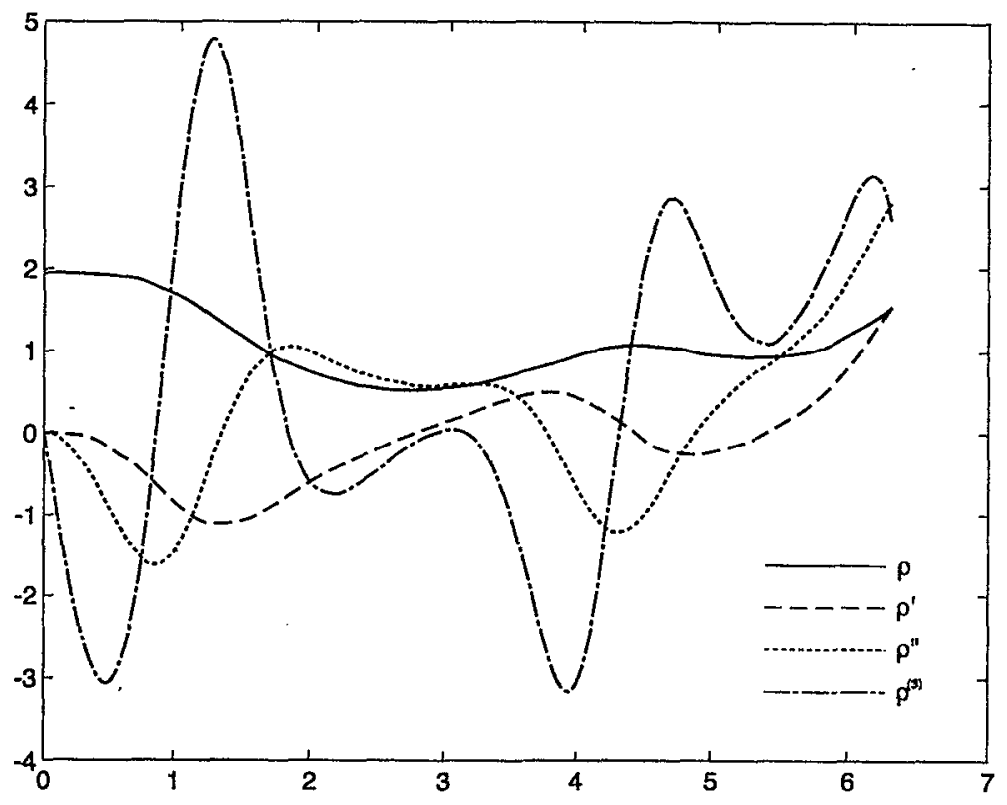

FIG. 4. An increase in $\rho^{\prime \prime}(0)$.

$$
G=\frac{1}{15}\left(\frac{3}{2}\right)^{2 / 3} a^{-2 / 3} b^{-2 / 3} .
$$

The graphs in Figs. 2-4 represent solutions to Eq. (5.3) computed by Matlab with a fourthand fifth-order Runge-Kuta-Fehlberg solver and an error tolerance set at 0.001 (the extra digit is reported for roundoff). In Figs. $2-4 \rho$ is represented by a solid line, $\rho^{\prime}$ by a dashed line, $\rho^{\prime \prime}$ by a dotted line, and $\rho^{(3)}$ by a line of dashes and dots.

Figure 2 corresponds to $\rho(0)=1.9531, \rho^{\prime}(0)=0, \rho^{\prime \prime}(0)=-8.44573974609$, and $\rho^{(3)}(0)=0$ and the (periodic) solution is an ellipse with parameters $a=1.25, b=0.8$. Figure 3 corresponds to $\rho(0)=1.9531, \rho^{\prime}(0)=0, \rho^{\prime \prime}(0)=-8.44573974609$, and $\rho^{(3)}(0)=5$. For this case $\rho(2 \pi)=1.9531$, $\rho^{\prime}(2 \pi)=-0.5187, \rho^{\prime \prime}(2 \pi)=-8.2622$, and $\rho^{(3)}(2 \pi)=18.2086$. Figure 4 corresponds to $\rho(0)$ $=1.9531, \rho^{\prime}(0)=0, \rho^{\prime \prime}(0)=0$, and $\rho^{(3)}(0)=0$. For this case $\rho(2 \pi)=1.6130, \rho^{\prime}(2 \pi)=1.6053$, $\rho^{\prime \prime}(2 \pi)=2.8857$, and $\rho^{(3)}(2 \pi)=2.5541$.

\section{ACKNOWLEDGMENT}

The author thanks Eugene Gutkin for suggesting that he examine invariant circles for the outer billiard map.

'J. Moser, Stable and Random Motions in Dynamical Systems, Ann. Math. Studies 77 (Princeton University, Princeton, NJ, 1973).

${ }^{2}$ S. Tabachnikov, "Commuting dual billiard maps," ETH preprint (1993).

${ }^{3}$ V. F. Lazutkin, "Existence of a continuum of closed invariant curves for a convex billiard," Math. USSR Izv. 7, (1), 185-214 (1973).

${ }^{4}$ E. Y. Amiran, "Caustics and evolutes for convex planar domains," J. Diff. Geom. 28, 345-357 (1988).

${ }^{5} \mathrm{~J}$. Moser, "On invariant curves of area preserving mappings of an annulus," Nachr. Akad. Wiss. Götingen Math. Phys. 1962, $1-20$.

${ }^{6} \mathrm{H}$. Poincaré, "Ŝ̉ur un théorèm de géométrie," Rand. Circ. Matemat. Palermo 33, 375-407 (1912)

7A. Denjoy, "Ŝur les courbes définies par les équations différentielles á le surface de tore," J. Math. Pure. Appliq. 11, 333-375 (1932).

${ }^{8}$ E. Gutkin and A. Katok, "Caustics for inner and outer billiards," Report No. PM 182 (November 1994), Penn State University. 